\title{
Simultaneous Packing and Covering in Sequence Spaces
}

\author{
Konrad J. Swanepoel
}

Received: 27 June 2008 / Revised: 4 March 2009 / Accepted: 8 April 2009 /

Published online: 7 May 2009

(C) Springer Science+Business Media, LLC 2009

\begin{abstract}
We adapt a construction of Klee (1981) to find a packing of unit balls in $\ell_{p}(1 \leq p<\infty)$ which is efficient in the sense that enlarging the radius of each ball to any $R>2^{1-1 / p}$ covers the whole space. We show that the value $2^{1-1 / p}$ is optimal.
\end{abstract}

Keywords Packings - Coverings - Simultaneous packing and covering constant . $\ell_{p}$ sequence space $\cdot$ Closest packing $\cdot$ Loosest covering

\section{Introduction}

The so-called simultaneous packing and covering constant of a convex body $C$ in Euclidean space is a certain measure of the efficiency of a packing or a covering by translates of $C$. This notion was introduced in various equivalent forms by Rogers [14], Ryškov [16], and L. Fejes Tóth [8] and in the lattice case can be traced back to Delone [5]. Its study has recently been given renewed attention by Zong [19-23] and others [9, 17]. Important contributions to the non-lattice case have also been made by Linhardt [13], Böröczky [2], and Doyle, Lagarias, and Randall [7]. Since this notion avoids the use of density, it can be used to study packings and coverings in hyperbolic spaces or infinite-dimensional spaces. Rogers [15] considered the infinite-dimensional lattice case for the unit balls of general Banach spaces. In this paper we determine the exact value of this constant for the $\ell_{p}$ spaces where $1 \leq p<\infty$. The main ingredient in the proof is an adaptation of a construction of Klee [10].

Dedicated to the memory of Victor Klee.

K.J. Swanepoel ( $₫)$

Fakultät für Mathematik, Technische Universität Chemnitz, 09107 Chemnitz, Germany

e-mail: konrad.swanepoel@gmail.com 


\section{The Simultaneous Packing and Covering Constant}

Let $(X,\|\cdot\|)$ be any normed space. Denote by $B(x, r)$ the closed ball with center $x \in X$ and radius $r$. A subset $P \subseteq X$ is (the set of centers of) an r-packing if the collection of balls $\{B(x, r): x \in P\}$ are pairwise disjoint. Equivalently, $P$ is $2 r$-dispersed, i.e., $d(x, y)>2 r$ for all distinct $x, y \in P$. For any $P \subseteq X$, define

$$
r(P):=\sup \{r: P \text { is an } r \text {-packing }\} .
$$

A subset $P \subseteq X$ is (the set of centers of) an $R$-covering (or $R$-net) if the collection of balls $\{B(x, R): x \in P\}$ cover $X$, i.e., $X=\bigcup_{x \in P} B(x, R)$. For any $P \subseteq X$, define

$$
R(P):=\inf \{R: P \text { is an } R \text {-covering }\} .
$$

Then $R(P)$ is the supremum of the radii of balls disjoint from $P$ :

$$
R(P)=\sup \{R \text { : for some } x \in X, B(x, R) \cap P=\emptyset\} .
$$

If $P$ is an $r$-packing, then $R(P)-r$ is the supremum of the radii of balls that are disjoint from $\bigcup_{p \in P} B(p, r)$, and if $P$ is an $R$-covering, then $R-r(P)$ is the supremum of the radii of balls that are contained in more than one of $B(p, R), p \in P$ [8].

Definition The simultaneous packing and covering constant of (the unit ball of) $X$ is

$$
\gamma(X):=\inf \{R(P): P \text { is a 1-packing }\} .
$$

We could also use 1-coverings to define this constant, as shown by the identity

$$
\gamma(X)^{-1}=\sup \{r(P): P \text { is a } 1 \text {-covering }\} .
$$

It is clear that $R(P) \geq 1$ for any 1-packing $P$. By Zorn's lemma there always exists a maximal 1 -packing, which is necessarily a 2-covering. Therefore,

$$
1 \leq \gamma(X) \leq 2
$$

If $X$ is finite-dimensional, then $\gamma(X)$ is exactly the simultaneous packing and covering constant of the unit ball of $X$, as discussed in the introduction.

\section{The Main Theorem}

The main result of the paper concerns the case $X=\ell_{p}, 1 \leq p<\infty$, which we recall is the space of real sequences $x=\left(x_{i}\right)_{i \in \mathbb{N}}$ such that $\sum_{i=1}^{\infty}\left|x_{i}\right|^{p}<\infty$ with norm

$$
\|x\|_{p}:=\left(\sum_{i=1}^{\infty}\left|x_{i}\right|^{p}\right)^{1 / p} .
$$


Theorem For each $p \in[1, \infty), \gamma\left(\ell_{p}\right)=2^{1-1 / p}$.

In particular, if $p$ is close to 1 , then $\gamma\left(\ell_{p}\right)$ is close to 1 , which means that there are very good packings of unit balls in $\ell_{p}$. Perhaps more surprisingly, if $p$ is very large, $\gamma\left(\ell_{p}\right)$ is close to 2 , i.e., any packing by unit balls has large holes.

In the next section we use a result of Burlak, Rankin, and Robertson [3] to show the lower bound $\gamma\left(\ell_{p}\right) \geq 2^{1-1 / p}$. It is more difficult to find good packings. In Sect. 5 we adapt a construction of Klee [10] to give packings that demonstrate the upper bound $\gamma\left(\ell_{p}\right) \leq 2^{1-1 / p}$. In fact, Klee already obtained this bound for $\ell_{p}(\kappa)$, where $\kappa$ is a regular cardinal such that $\kappa^{\aleph_{0}}=\kappa$. In our case, $\kappa=\aleph_{0}$, and then his construction has to be modified substantially.

\section{The Lower Bound}

For a proof of the following packing property of $\ell_{p}$, see $[3,11]$, or [18].

Lemma 1 If the unit ball of $\ell_{p}$ contains an infinite $\alpha$-dispersed set, then $\alpha \leq 2^{1 / p}$.

To prove $\gamma\left(\ell_{p}\right) \geq 2^{1-1 / p}$, it is sufficient to show the following:

Proposition 2 Let $P$ be a 1-dispersed subset of $\ell_{p}$, where $1 \leq p<\infty$. Then $R(P) \geq$ $2^{-1 / p}$.

Proof Let $0<\varepsilon<R(P)$. Set $r:=R(P)-\varepsilon$ and $\delta:=\left((r+2 \varepsilon)^{p}-r^{p}\right)^{1 / p}$. By (1) there exists $c \in \ell_{p}$ such that $B(c, r) \cap P=\emptyset$. Translate $P$ by $-c$ so that we may assume without loss of generality that $c=o$. Thus $\|x\|>r$ for all $x \in P$.

We claim that $Q:=B(o, r+\delta+2 \varepsilon) \cap P$ is infinite. Suppose to the contrary that $Q$ is finite. As usual, we denote by $e_{n}$ the sequence which is 1 in position $n$ and 0 in all other positions. For any $n \in \mathbb{N}$ and $x \in Q$,

$$
\left\|x-\delta e_{n}\right\|_{p}^{p}=\|x\|_{p}^{p}-\left|x_{n}\right|^{p}+\left|x_{n}-\delta\right|^{p} .
$$

Therefore,

$$
\lim _{n \rightarrow \infty}\left\|x-\delta e_{n}\right\|_{p}^{p}=\|x\|_{p}^{p}+\delta^{p}>r^{p}+\delta^{p}=(r+2 \varepsilon)^{p} .
$$

Since $Q$ is finite, there exists $n \in \mathbb{N}$ such that for all $x \in Q,\left\|x-\delta e_{n}\right\|>r+2 \varepsilon$. On the other hand, for any $x \in P \backslash Q,\|x\|>r+\delta+2 \varepsilon$, and then the triangle inequality gives $\left\|x-\delta e_{n}\right\|>r+2 \varepsilon$. Therefore, $B\left(\delta e_{n}, r+2 \varepsilon\right) \cap P=\emptyset$, which gives $R(P) \geq r+2 \varepsilon$, a contradiction.

Thus $Q$ is infinite, and by Lemma 1 ,

$$
R(P)+\delta+\varepsilon=r+\delta+2 \varepsilon \geq 2^{-1 / p} .
$$

By letting $\varepsilon \rightarrow 0$ we obtain $R(P) \geq 2^{-1 / p}$, as required. 


\section{Constructing an Optimal Packing}

To prove the upper bound $\gamma\left(\ell_{p}\right) \leq 2^{1-1 / p}$, it is sufficient to show the following:

Proposition 3 For any $1 \leq p<\infty$, there exists a $2^{1 / p}$-dispersed set $P \subseteq \ell_{p}$ such that $R(P) \leq 1$.

Proof We recursively construct the set $P$ together with the space, which in the end is isometric to $\ell_{p}$.

If $A$ is any set, we denote by $\ell_{p}(A)$ the normed space of all real-valued functions $f$ on $A$ with countable support $\operatorname{supp}(f):=\{a \in A: f(a) \neq 0\}$ such that $\sum_{a \in \operatorname{supp}(f)}|f(a)|^{p}<\infty$, with norm

$$
\|f\|_{p}:=\left(\sum_{a \in \operatorname{supp}(f)}|f(a)|^{p}\right)^{1 / p} .
$$

Thus $\ell_{p}=\ell_{p}(\mathbb{N})$ is isometric to $\ell_{p}(A)$ if $A$ is countably infinite. For any $a \in A$, let $e_{a}$ be the function on $A$ such that $e_{a}(a)=1$ and $e_{a}(b)=0$ for all $b \in A, b \neq a$. If $A \subseteq A^{\prime}$, then we consider $\ell_{p}(A)$ to be a subspace of $\ell_{p}\left(A^{\prime}\right)$ in the natural way.

We construct two sequences of countable sets $P_{n}$ and $D_{n}$. Let $P_{1}=\emptyset$ and $D_{1}=$ $\{0\}=\ell_{p}(\emptyset)$. If $P_{1}, \ldots, P_{n}$ and $D_{1}, \ldots, D_{n}$ have been constructed for some $n \geq 1$, let

$$
P_{n+1}:=\left\{x+e_{x}: x \in D_{n}\right\} \subseteq \ell_{p}\left(\bigcup_{i=1}^{n} D_{i}\right),
$$

and let $D_{n+1}$ be a countable dense subset of

$$
\ell_{p}\left(\bigcup_{i=1}^{n} D_{i}\right) \bigcup \bigcup\left\{B(x, 1): x \in \bigcup_{i=1}^{n+1} P_{i}\right\} .
$$

By the definition of $P_{n+1}$ it follows that $D_{k} \subseteq \bigcup_{x \in P_{k+1}} B(x, 1)$ for each $k=1, \ldots, n$; hence, $D_{n+1}$ is disjoint from $\bigcup_{i=1}^{n} D_{i}$. It follows that the $P_{n}$ are also pairwise disjoint.

Let $P:=\bigcup_{n \in \mathbb{N}} P_{n}$. Then $P$ is a subset of the space $\ell_{p}\left(\bigcup_{n \in \mathbb{N}} D_{n}\right)$, which is isometric to $\ell_{p}$ (note that already $D_{2}$ is infinite). We now show that $P$ is $2^{1 / p}$-dispersed and is a $(1+\varepsilon)$-covering for all $\varepsilon>0$.

Choose two arbitrary elements $x+e_{x}, y+e_{y} \in P$, where $x \in D_{n}$ and $y \in D_{m}$, $x \neq y$, and $n \leq m$. Since $\operatorname{supp}(x), \operatorname{supp}(y) \subseteq \bigcup_{i=1}^{m-1} D_{i}$, which is disjoint from $D_{m}$, it follows that $\operatorname{supp}(x-y)$ and $\operatorname{supp}\left(e_{y}\right)=\{y\}$ are disjoint. We distinguish between two cases.

If $n=m$, then $\operatorname{supp}\left(e_{x}\right)=\{x\}$ is also disjoint from $\operatorname{supp}(x-y)$ and $\operatorname{supp}\left(e_{y}\right)$; hence,

$$
\left\|\left(x+e_{x}\right)-\left(y+e_{y}\right)\right\|_{p}^{p}=\left\|x-y+e_{x}-e_{y}\right\|_{p}^{p}=\|x-y\|_{p}^{p}+1+1>2 .
$$

In the second case, $n<m$. Since $y \in D_{m}$ and $x+e_{x} \in P_{n+1}$, it follows that $y \notin$ $B\left(x+e_{x}, 1\right)$; hence, $\left\|x+e_{x}-y\right\|_{p}^{p}>1$. Since $\operatorname{supp}\left(x+e_{x}-y\right)$ and $\operatorname{supp}\left(e_{y}\right)$ are 
now disjoint,

$$
\left\|\left(x+e_{x}\right)-\left(y+e_{y}\right)\right\|_{p}^{p}=1+\left\|x+e_{x}-y\right\|_{p}^{p}>1+1 .
$$

It follows that $P$ is $2^{1 / p}$-dispersed.

Let $\varepsilon>0$ and choose an arbitrary $x \in \ell_{p}\left(\bigcup_{n \in \mathbb{N}} D_{n}\right)$. Choose $N \in \mathbb{N}$ large enough such that $\|x-y\|_{p}<\varepsilon / 2$ for some $y \in \ell_{p}\left(\bigcup_{i=1}^{N-1} D_{i}\right)$. If $y \in \bigcup\{B(z, 1): z \in$ $\left.\bigcup_{i=1}^{N} P_{i}\right\}$, then for some $z \in \bigcup_{i=1}^{N} P_{i}$,

$$
\|x-z\|_{p} \leq\|x-y\|_{p}+\|y-z\|_{p}<1+\varepsilon / 2 .
$$

If, on the other hand, $y \notin \bigcup\left\{B(z, 1): z \in \bigcup_{i=1}^{N} P_{i}\right\}$, then there exists $z \in D_{N}$ such that $\|y-z\|_{p}^{p}<(1+\varepsilon / 2)^{p}-1$. Note that $z+e_{z} \in P_{N+1}$. Since $z \in D_{N}$ and $y, z \in$ $\ell_{p}\left(\bigcup_{i=1}^{N} D_{i}\right), \operatorname{supp}\left(e_{z}\right)=\{z\}$ is disjoint from $\operatorname{supp}(y-z)$. Thus,

$$
\left\|z+e_{z}-y\right\|_{p}^{p}=1+\|z-y\|_{p}^{p}<(1+\varepsilon / 2)^{p},
$$

and by the triangle inequality, $\left\|z+e_{z}-x\right\|<1+\varepsilon$. It follows that $P$ is a $(1+\varepsilon)$ covering.

\section{Closing Remarks and Questions}

6.1

Define the lattice simultaneous packing and covering constant of (the unit ball of) a normed space $X$ to be

$$
\gamma^{*}(X):=\inf \{R(P): P \text { is a 1-packing and a subgroup of }(X,+)\} .
$$

Since an arbitrary maximal 1-packing cannot be expected to be closed under addition, it becomes nontrivial to find an upper bound to $\gamma^{*}(X)$. Rogers [15] showed that $\gamma^{*}(X) \leq 3$ for any Banach space $X$. Can this upper bound be improved to $\gamma^{*}(X) \leq 2$ ?

6.2

Let

$$
\beta(X):=\sup \{\alpha: \text { the unit ball of } X \text { contains an infinite } \alpha \text {-dispersed set }\} .
$$

This is also known as the separation measure of noncompactness of the unit ball of $X$ [1]. Elton and Odell proved that $\beta(X)>1$ for any Banach space $X$. For more information, see [12] and references therein. Using a result of Corson [6], Casini, Papini, and Zanco [4] generalized Proposition 2 by showing that $\gamma(X) \geq 2 / \beta(X)$ if $X$ has an infinite-dimensional reflexive subspace. This, together with Lemma 1 , immediately implies Proposition 2. However, our proof for $\ell_{p}$ is direct.

The following question is essentially stated in [4]: Is $\gamma(X)=2 / \beta(X)$ or perhaps even $\gamma^{*}(X)=2 / \beta(X)$ ? 


\section{References}

1. Ayerbe Toledano, J.M., Domínguez Benavides, T., López Acedo, G.: Measures of Non-Compactness in Metric Fixed Point Theory. Birkhäuser, Basel (1997)

2. Böröczky, K.: Closest packing and loosest covering of the space with balls. Stud. Sci. Math. Hung. 21, 79-89 (1986)

3. Burlak, J.A.C., Rankin, R.A., Robertson, A.P.: The packing of spheres in the space $\ell_{p}$. Proc. Glasg. Math. Assoc. 4, 22-25 (1958)

4. Casini, E., Papini, P.L., Zanco, C.: Separation and Approximation in Normed Linear Spaces. International Series of Numerical Mathematics, vol. 76. Birkhäuser, Basel (1986)

5. Delone, B.N.: Geometry of positive quadratic forms. Usp. Mat. Nauk 3, 16-62 (1937)

6. Corson, H.H.: Collections of convex sets which cover a Banach space. Fund. Math. 49, 143-145 (1961)

7. Doyle, P.G., Lagarias, J.C., Randall, D.: Self-packing of centrally symmetric convex bodies in $\mathbf{R}^{2}$. Discrete Comput. Geom. 8, 171-189 (1992)

8. Fejes Tóth, L.: Close packing and loose covering with balls. Publ. Math. Debr. 23, 323-326 (1976)

9. Henk, M.: Free planes in lattice sphere packings. Adv. Geom. 5, 137-144 (2005)

10. Klee, V.: Dispersed Chebyshev sets and coverings by balls. Math. Ann. 257, 251-260 (1981)

11. Kottman, C.A.: Packing and reflexivity in Banach spaces. Trans. Am. Math. Soc. 150, 565-576 (1970)

12. Kryczka, A., Prus, S.: Separated sequences in nonreflexive Banach spaces. Proc. Am. Math. Soc. 129, 155-163 (2000)

13. Linhart, J.: Closest packings and closest coverings by translates of a convex disc. Stud. Sci. Math. Hung. 13, 157-162 (1978)

14. Rogers, C.A.: A note on coverings and packings. J. Lond. Math. Soc. 25, 327-331 (1950)

15. Rogers, C.A.: Lattices in Banach spaces. Mitt. Math. Sem. Giessen 165, 155-167 (1984)

16. Ryškov, S.S.: Density of an $(r, R)$-system. Mat. Zametki 16, 447-454 (1974)

17. Schürmann, A., Vallentin, F.: Computational approaches to lattice packing and covering problems. Discrete Comput. Geom. 35, 73-116 (2006)

18. Wells, J.H., Williams, L.R.: Embeddings and Extensions in Analysis. Springer, New York (1975)

19. Zong, C.: From deep holes to free planes. Bull. Am. Math. Soc. (N.S.) 39, 533-555 (2002)

20. Zong, C.: Simultaneous packing and covering in the Euclidean plane. Monatsh. Math. 134, 247-255 (2002)

21. Zong, C.: Simultaneous packing and covering of centrally symmetric convex bodies. In: IV International Conference in Stochastic Geometry, Convex Bodies, Empirical Measures \& Applications to Engineering Science, vol. II, Tropea, 2001. Rend. Circ. Mat. Palermo (2) Suppl. 70, part II, 387-396 (2002)

22. Zong, C.: Simultaneous packing and covering in three-dimensional Euclidean space. J. Lond. Math. Soc. (2) 67, 29-40 (2003)

23. Zong, C.: The simultaneous packing and covering constants in the plane. Adv. Math. 218, 653-672 (2008) 